\title{
EXPONENTIAL STABILITY AND UNSTABILITY OF SEMIGROUPS OF LINEAR OPERATORS IN BANACH SPACES
}

\author{
M. Megan, A. L. Sasu, B. Sasu, A. Pogan
}

\begin{abstract}
Necessary and sufficient conditions for uniform exponential stability and uniform exponential unstability of semigroups of linear operators are given, in terms of Banach function spaces and Banach sequence spaces, respectively. We generalize some well-known theorems proved by Datko, Pazy, Rolewicz and Neerven concerning the exponential stability of $C_{0}$ semigroups. We shall obtain the versions of the theorems mentioned above, for the case of uniform exponential unstability of $C_{0}$-semigroups.
\end{abstract}

Mathematics subject classification (2000): 34D05, 34D20, 47D06.

Key words and phrases: Semigroup, uniform exponential stability, uniform exponential unstability, Banach function spaces, Banach sequence spaces.

\section{REFERENCES}

[1] R. DATKO, Uniform asymptotic stability of evolutionary processes in Banach spaces, SIAM J. Math. Anal. 3 (1972), 428-445.

[2] R. DATKO, Extending a theorem of Liapunov to Hilbert spaces, J. Math. Anal. Appl. 32 (1970), 610-616.

[3] K. J. ENGEL, R. NAGEL, One-parameter Semigroups for Linear Evolution Equations, Graduate Texts in Mathematics, 194, Berlin (2000).

[4] W. LitTMan, A generalization of the theorem Datko-Pazy, Lecture Notes in Control and Inform. Sci. 130 (1983), 318-323.

[5] M. Megan, A. L. SASu, B. SASu, On uniform exponential stability of periodic evolution operators in Banach spaces, Acta Math. Univ. Comenian. 69 (2000), 97-106.

[6] M. Megan, B. Sasu, A. L. Sasu, On uniform exponential stability of evolution families, Riv. Matem. Univ. Parma 4 (2001), 27-43.

[7] M. Megan, A. L. Sasu, B. Sasu, On uniform exponential stability of linear skew-product semiflows in Banach spaces, Bull. Belg. Math. Soc. Simon Stevin 9 (2002).

[8] M. Megan, A. L. Sasu, B. SASU, A discrete variant of the theorem of Littman, Proceed. Conf. Analysis, Funct. Eq. Approx. Theory and Convexity, Cluj-Napoca (1999), 282-290.

[9] M. Megan, A. Pogan, On a generalization of a theorem of Datko and Pazy, Proceed. Conf. Analysis, Funct. Eq. Approx. Theory and Convexity, Cluj-Napoca (1999), 164-170.

[10] M. MEgan, A. PogAn, On a theorem of Rolewicz for semigroups of operators in locally convex spaces, Ann. Math. Blaise Pascal 7 (2000), 23-35.

[11] M. Megan, A. Pogan, On exponential stability of semigroups of linear operators in Banach spaces, Seminar Math. Anal. Appl. Control Theory 124 (2001).

[12] P. MeYER-NieBERG, Banach Lattices, Springer Verlag, Berlin, Heidelberg, New York, 1991.

[13] J. van NEERVEN, The Asymptotic Behaviour of Semigroups of Linear Operators, Birkhäuser, 1995.

[14] J. VAN NEERVEN, Exponential stability of operators and semigroups, J. Func. Anal. 130 (1995), 293-309.

[15] J. VAN NEERVEN, Lower semicontinuity and the theorem of Datko and Pazy, preprint, University of Karlsruhe (2000).

[16] A. PAZY, Semigroups of Linear Operators and Applications to Partial Differential Equations, Springer-Verlag, Berlin, Heidelberg, New-York, 1983. 
[17] A. PAZY, On the applicability of Liapunov's theorem in Hilbert spaces, SIAM. J. Math. Anal. Appl. 3 (1972), 291-294.

[18] S. RolewicZ,, On uniform N-equistability, J. Math. Anal. Appl. 115 (1986), 434-441.

[19] Z. ZABCZYK, Remarks on the control of discrete-time distributed parameter systems, SIAM J. Control Optim. 12 (1974), 721-735.

[20] Q. ZHENG, The exponential stability and the perturbation problem of linear evolution systems in Banach spaces, J. Sichuan Univ. 25 (1988), 401-411. 